\title{
Advances in the treatment of Hodgkin lymphoma
}

\author{
Dennis A. Eichenauer • Andreas Engert
}

Received: 26 September 2012/Accepted: 30 September 2012/Published online: 11 October 2012

(c) The Japanese Society of Hematology 2012

\begin{abstract}
In the past decades, Hodgkin lymphoma (HL) has turned from an incurable disease to one with the most favorable prognosis among adult malignancies. This is due to the introduction of effective multi-agent chemotherapy and the optimization of radiation techniques. At present, 80-90\% of patients achieve long-term remission when receiving appropriate first-line treatment. Even in case of relapse, up to $50 \%$ can be successfully salvaged with highdose chemotherapy and autologous stem cell transplantation. Thus, current studies do not necessarily aim at increasing treatment efficacy but rather focus on a possible reduction of acute and late toxicity by decreasing treatment intensity if possible. One promising strategy to spare therapy in good-risk patients is early response-adapted treatment stratification according to the result of interim positron emission tomography. The evaluation of novel drugs and the optimization of treatment for elderly HL patients and those with nodular lymphocyte-predominant $\mathrm{HL}$ are further aspects that are currently being addressed in ongoing trials. This review will give an overview on more recent clinical trials and discuss possible future strategies for the treatment of HL.
\end{abstract}

Keywords Hodgkin lymphoma - Therapy - Novel drugs

D. A. Eichenauer · A. Engert $(\bowtie)$

First Department of Internal Medicine, University Hospital Cologne, 50937 Cologne, Germany

e-mail: a.engert@uni-koeln.de

D. A. Eichenauer · A. Engert

German Hodgkin Study Group (GHSG), Cologne, Germany

\section{Introduction}

Hodgkin lymphoma (HL) is a B cell-derived malignancy of the lymphatic system mainly affecting young adults in their third and fourth decade of life. The disease commonly presents with indolent lymphadenopathy and can be accompanied by B-symptoms (i.e., undulating fever $>38^{\circ} \mathrm{C}$, drenching night sweats, unintended weight loss $>10 \%$ ). Staging procedures include computed tomography (CT) of neck, chest and abdomen, bone marrow biopsy, organ function tests and laboratory tests. Thereafter, patients are allocated to different risk groups (early favorable, early unfavorable, advanced) according to their clinical stage and the presence or absence of adverse clinical features (Table 1). The latter include involvement of three or more nodal areas, elevated erythrocyte sedimentation rate (ESR), large mediastinal mass of more than one-third of maximum chest diameter and extranodal disease [1]. Treatment of HL consists of chemotherapy and/or radiotherapy (RT). Patients with early stages are usually treated with a brief chemotherapy followed by involved-field RT (IF-RT) while patients with more advanced stages receive aggressive chemotherapy optionally followed by localized RT. More than $80 \%$ of patients can be cured with these approaches. However, there are still areas of unmet medical need. For instance, patients diagnosed with their first relapse can be successfully rescued in about $50 \%$ of cases. In contrast, cure is rare among patients with multiple relapses and their situation is commonly palliative. Furthermore, patients older than 60 years have a significantly worse clinical outcome than younger patients. Thus, novel targeted drugs and new treatment strategies for these patients are needed. This review summarizes recent key publications defining the present standard of care in the treatment of HL and gives an overview on the current efforts to optimize HL treatment. 
Table 1 Definition of risk groups by the EORTC/GELA and the GHSG

\begin{tabular}{lll}
\hline $\begin{array}{l}\text { Treatment } \\
\text { group }\end{array}$ & EORTC/GELA & GHSG \\
\hline $\begin{array}{l}\text { Early } \\
\text { favorable }\end{array}$ & $\begin{array}{c}\text { CS I-II without risk factors } \\
\text { (supradiaphragmatic) }\end{array}$ & CS I-II without risk factors \\
$\begin{array}{c}\text { Early } \\
\text { unfavorable }\end{array}$ & $\begin{array}{l}\text { CS I-II with } \geq 1 \text { risk factors } \\
\text { (supradiaphragmatic) }\end{array}$ & $\begin{array}{c}\text { CS I, CS IIA with } \geq 1 \text { risk factors; CS IIB with risk } \\
\text { factors C/D, but not A/B }\end{array}$ \\
$\begin{array}{l}\text { Advanced } \\
\text { CS III-IV }\end{array}$ & CS IIB with risk factors A/B, CS III/IV \\
& (A) Large mediastinal mass & (A) Large mediastinal mass \\
& (B) Age $\geq 50$ years & (B) Extranodal disease \\
& (C) Elevated ESR & (C) Elevated ESR \\
& (D) $\geq 4$ nodal areas & (D) $\geq 3$ nodal areas \\
\hline
\end{tabular}

\section{Treatment of early favorable HL}

For decades, standard treatment for patients with early favorable HL (i.e., patients with stage I/II disease without clinical risk factors) was RT alone. More than $90 \%$ of patients achieved complete remission (CR) with this approach, but relapse rate was unacceptably high with $30 \%$ and more. Thus, groups such as the German Hodgkin Study Group (GHSG) and the European Organisation for Research and Treatment of Cancer (EORTC) conducted randomized clinical trials comparing RT alone with combined-modality strategies with the aim to improve the clinical outcome of patients with early favorable HL.

In the GHSG HD7 trial, patients were randomly assigned to receive either $30 \mathrm{~Gy}$ extended-field RT (EFRT) alone or two cycles of ABVD (adriamycin, bleomycin, vinblastine, dacarbazine) followed by the same RT [2]. Response rates did not differ between treatment arms. However, at a median follow-up of 87 months, the 7-year freedom from treatment failure (FFTF) rate was significantly better in the combined-modality arm (88 vs. $67 \%$ ). The advantage in tumor control had not yet translated into a superior overall survival (OS) (94 vs. $92 \%$ ) at 7 years and future analyses with longer follow-up are required for a definite answer to this question.

The results of the randomized EORTC H8F trial were similar. Treatment arms consisted of three cycles of MOPP (mechlorethamine, vincristine, procarbazine, prednisone)/ ABV followed by IF-RT or subtotal nodal irradiation (STNI) alone [3]. With a median observation of 92 months, patients receiving combined-modality treatment had a significantly superior 5-year event-free survival (EFS) (98 vs. $74 \%$ ) and-in contrast to the GHSG HD7 trial-also better 10-year OS estimates (97 vs. $92 \%$ ).

On the basis of these two large controlled randomized trials, combined-modality treatment consisting of a brief chemotherapy followed by IF-RT was adopted as standard of care for the treatment of early favorable HL by most institutions worldwide.
In the randomized GHSG follow-up study (HD10), 1370 patients received either two or four cycles of ABVD followed by IF-RT at 20 or $30 \mathrm{~Gy}$. At a median follow-up of 90 months, FFTF and OS rates exceeded 90 and $95 \%$, respectively, irrespective of the treatment arm (Fig. 1). Thus, the least toxic approach consisting of two cycles of ABVD followed by 20 Gy IF-RT has been adopted as standard of care within the GHSG and an increasing number of centers worldwide [4].

The more recent HD13 trial aimed at detoxifying treatment by reducing the number of drugs given. Patients were randomized between two cycles of ABVD, ABV, AVD or AV chemotherapy followed by 30 Gy IF-RT [5]. Both, the ABV and the AV arm were closed prematurely since tumor control with these regimens was significantly worse than with ABVD. The 4-year FFTF rates were 84.5 and $75.3 \%$ with $\mathrm{ABV}$ and $\mathrm{AV}$, respectively, compared with a 4-year FFTF beyond $90 \%$ with ABVD; OS at 4 years did not differ between treatment arms. Thus, dacarbazine appears to be an essential component in the ABVD protocol when used in early favorable HL. The question whether AVD and ABVD are equally effective cannot be answered to date and needs further follow-up.

Ongoing trials such as the GHSG HD16 trial and the EORTC H10F study evaluate interim positron emission tomography (PET) for treatment stratification. In the randomized HD16 trial (NCT00736320), all patients initially receive two cycles of ABVD before interim PET is performed. Then, patients in the standard arm have 20 Gy IFRT irrespective of their PET result. In the experimental arm, 20 Gy IF-RT is only applied to patients with an incomplete metabolic response while patients with a negative PET are not irradiated. In the H10F trial (NCT00433433) conducted by the EORTC and the Groupe d'Etude des Lymphomes de l'Adulte (GELA), treatment in the experimental arm is also stratified according to the result of an interim PET performed after two cycles of ABVD. While patients assigned to the standard arm receive a total of three cycles of ABVD followed by involved-node 


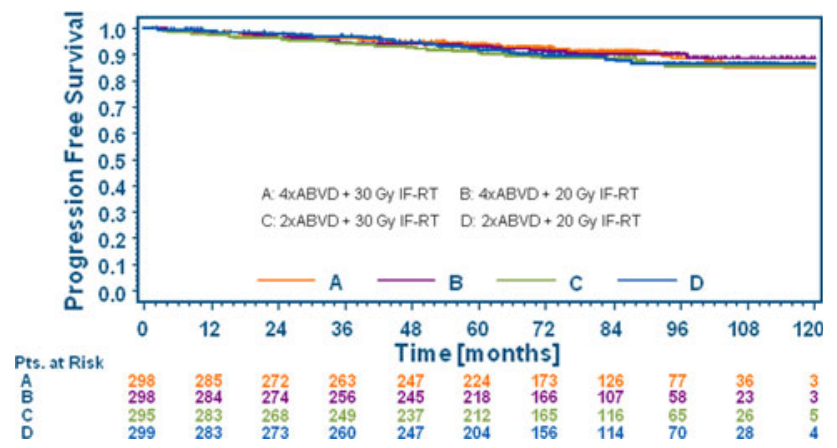

Fig. 1 Progression-free survival curves for patients within the HD10 trial for early favorable $\mathrm{HL}$ according to treatment arm $(A 4 \times \mathrm{ABVD}+30$ Gy IF-RT, $B 4 \times \mathrm{ABVD}+20$ Gy IF-RT, $C 2 \times \mathrm{ABVD}+30$ Gy $\mathrm{IF}-\mathrm{RT}, \quad D 2 \times \mathrm{ABVD}+20$ Gy IF-RT) (adopted from Engert et al. [4])

RT (IN-RT) irrespective of the PET result, patients randomized to the experimental arm received two additional cycles of ABVD without additional irradiation in case of a complete metabolic response when the trial started recruitment; patients with a positive interim PET continued treatment with two cycles of escalated BEACOPP (bleomycin, etoposide, adriamycin, cyclophosphamide, vincristine, procarbazine, prednisone) followed by involved-node RT (IN-RT). However, after an interim analysis revealed an increased frequency of events among patients receiving chemotherapy only, this treatment arm was closed early so that all patients with a negative PET now receive consolidating IN-RT. Although longer follow-up is required for final conclusions, the premature closure of the chemotherapy-only arm underscores the fact that treatment stratification according to interim PET in patients with early favorable HL should be restricted to clinical trials.

In summary, a brief chemotherapy with ABVD followed by IF-RT represents a safe and highly active treatment that is widely being adopted as standard of care for patients with early favorable HL. The question whether treatment can be further reduced in order to decrease the risk of adverse effects such as lung failure or RT-associated secondary solid tumors will be answered by future analyses of recently completed and ongoing randomized clinical trials.

\section{Early unfavorable HL}

Combined-modality approaches consisting of four cycles of chemotherapy followed by IF-RT represent the standard of care for patients with early unfavorable HL (i.e., those with stage I/II disease presenting with clinical risk factors).

However, the optimal chemotherapy is still undefined.

In the EORTC H8U trial, three different regimens were randomly compared [3]. Patients were assigned to receive either six cycles of MOPP/ABV plus IF-RT, four cycles of
MOPP/ABV plus IF-RT or four cycles of MOPP/ABV plus STNI. There were no differences with respect to the 5-year EFS and the 10-year OS estimates. Thus, it was concluded that four courses of chemotherapy followed by IF-RT should be the standard of care for patients with early unfavorable HL.

In order to improve the tumor control in this patient group, the GHSG and the EORTC both incorporated BEA$\mathrm{COPP}_{\text {baseline }}$ into their next trial generation. In the GHSG HD11 trial, 1395 patients received either four cycles of

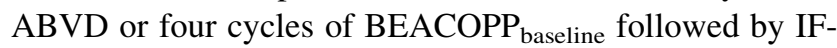
RT at 20 or 30 Gy [6]. Freedom from treatment failure with

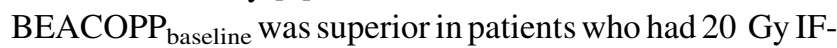
RT after chemotherapy while there were no differences between BEACOPP $_{\text {baseline }}$ and ABVD in patients who received 30 Gy IF-RT. Overall survival did not differ between the four treatment arms. Thus, BEACOPP ${ }_{\text {baseline }}$ was not adopted as novel standard chemotherapy regimen for patients with early unfavorable HL due to increased toxicity observed in comparison with ABVD. Preliminary results from the EORTC H9U trial were similar [7]. Within this trial, patients were randomized between six cycles of ABVD, four

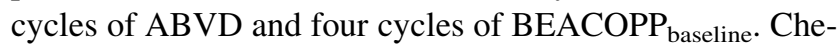
motherapy was followed by 30 Gy IF-RT. Event-free survival rates were comparable for patients from all treatment arms but increased toxicity was observed with BEA-

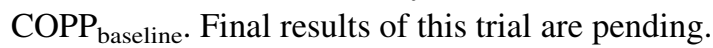

The GHSG follow-up HD14 trial further intensified

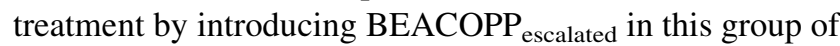
patients [8]. This regimen was originally developed for patients with advanced HL and is associated with more hematotoxicity necessitating the administration of granulocyte-colony stimulating factor (G-CSF) [9]. Patients enrolled in the HD14 trial had four cycles of ABVD followed by 30 Gy IF-RT or two cycles of BEACOPP ${ }_{\text {escalated }}$ followed by two cycles of ABVD (" $2+2$ ") and 30 Gy IFRT. The final analysis at a median follow-up of 43 months revealed a better tumor control with 5-year FFTF estimates of $94.8 \%$ for the " $2+2$ " protocol as compared with $87.7 \%$ for the standard arm. However, the advantage in terms of FFTF has not translated into a better OS so far. Thus, follow-up analyses of the HD14 trial especially addressing the question of long-term safety in the " $2+2$ " arm are needed for final conclusions.

Similar to early favorable HL, current trials including patients with early unfavorable $\mathrm{HL}$ such as the GHSG HD17 (NCT01356680) and the EORTC/GELA H10U (NCT00433433) trial aim at tailoring treatment according to the results of an interim PET. In the HD17 trial, all patients receive chemotherapy according to the " $2+2$ " regimen before a PET is performed. In the standard arm, patients receive additional 30 Gy IF-RT irrespective of the PET result. In the experimental arm, patients with a 
complete metabolic response do not receive further treatment while patients with residual PET-positive lymphoma have 30 Gy IN-RT. Aim of the study is to evaluate whether it is possible to spare RT in patients with a negative PET

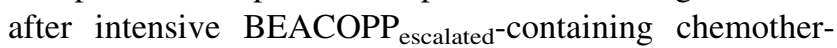
apy. The standard arm in the EORTC/GELA H10U trial consists of four cycles of ABVD followed by IN-RT irrespective of the result of an interim PET performed after the second chemotherapy cycle. In the experimental arm of the study, patients with a negative PET initially received a total of six cycles of ABVD without consolidating RT while patients with a positive PET continued treatment with two cycles of escalated BEACOPP before receiving IN-RT. However, the chemotherapy-only arm was closed on the basis of an interim analysis due to an increased number of events so that all patients with a negative PET now receive additional RT.

Long-term results from a trial conducted by the National Cancer Institute of Canada (NCIC) and the Eastern Cooperative Oncology Group (ECOG) indicate that chemotherapy-only approaches appear possible in patients with early unfavorable HL, at least in those with non-bulky disease [10]. The 276 patients with early HL presenting with unfavorable clinical features included in this trial were randomized to receive either $4-6$ cycles of ABVD alone or two cycles of ABVD followed by STNI. At a median follow-up of 11.3 years, freedom from disease progression was clearly better in the group receiving combined-modality treatment. However, this did not translate into a superior OS. In contrast, OS was better for patients treated with chemotherapy alone. This fact was mainly attributed to the increased number of deaths from secondary neoplasia among patients who had received combined-modality treatment. Nevertheless, one should keep in mind that the RT technique used in this trial is outdated and no longer used. In addition, five deaths in the combined-modality group were due to reasons very likely not associated with RT such as drowning, suicide and Alzheimer's disease while there was no death due to such reasons in the chemotherapy-only arm. In summary, chemotherapy only might represent a treatment option in patients with non-bulky early unfavorable HL refusing radiation but combined-modality should remain standard until further data from larger randomized trials supporting chemotherapy-only approaches for this patient group are available.

\section{Treatment of advanced stages}

Treatment of advanced HL usually consists of 6-8 cycles of chemotherapy followed by localized RT. The protocols
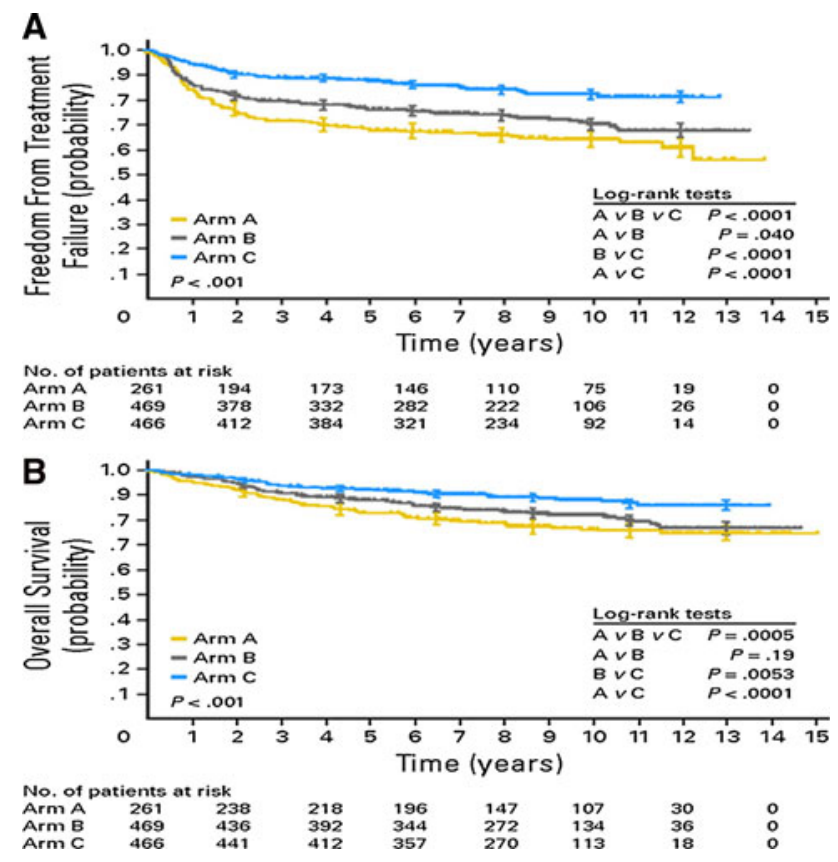

Fig. 2 Freedom from treatment failure (a) and overall survival (b) curves for patients within the HD9 trial for advanced HL according to treatment arm $(\mathbf{a} 8 \times \mathrm{COPP} / \mathrm{ABVD}$, b $8 \times \mathrm{BEA}$ $\mathrm{COPP}_{\text {baseline, }}$ c $8 \times \mathrm{BEACOPP}_{\text {escalated }}$ ) (adopted from Engert et al. [15])

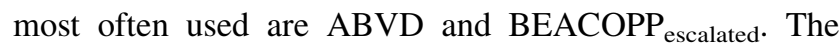
question whether one of these protocols may be more suitable for the treatment of advanced HL is an ongoing subject of debate since more than 10 years.

ABVD was shown to be equally effective and less toxic than MOPP and MOPP/ABVD in a randomized clinical trial by Cancer and Leukemia Group B (CALGB). With ABVD, MOPP and MOPP/ABVD, 5-year failure-free survival rates were 61,50 and $65 \%$, and 5-year OS rates were 73,66 and $75 \%$, respectively [11]. These results were confirmed by two follow-up analyses of the trial [12, 13]. Since MOPP-containing regimens are associated with a substantially higher risk for the development of adverse late effects, secondary leukemia in particular, 6-8 cycles of ABVD have been widely adopted as standard of care for the treatment of advanced HL.

With the aim of improving the treatment for patients with advanced HL, the dose-intensified BEACOPP escalated $_{\text {pro- }}$ tocol was developed by the GHSG. Within the pivotal HD9 trial, the novel regimen was randomly compared with a rapidly alternating combination of COPP (cyclophosphamide, vincristine, procarbazine, prednisone) and $\mathrm{ABVD}$ (COPP/ABVD), the standard at that time, and BEA$\mathrm{COPP}_{\text {baseline [14]. The final analysis revealed significantly }}$ better 5-year FFTF and OS rates for BEACOPP escalated $_{\text {as }}$ compared to COPP/ABVD and BEACOPP baseline $_{\text {(87 vs. } 69}$ 
Table 2 Causes of death within the HD15 trial for advanced HL according to treatment arm

\begin{tabular}{llll}
\hline & $8 \times$ BEACOPP $_{\text {escalated }}(\%)$ & $6 \times$ BEACOPP $_{\text {escalated }}(\%)$ & $8 \times$ BEACOPP-14 $(\%)$ \\
\hline HL & 1.8 & 1.5 & 2.1 \\
Tox first-line & 2.1 & 0.8 & 0.8 \\
Tox salvage & 0.3 & 0.3 & 0.1 \\
2nd neoplasia & 1.8 & 0.7 & 1.1 \\
Others & 1.3 & 1.2 & 0.9 \\
Overall & 7.5 & 4.6 & 5.2 \\
\hline
\end{tabular}

vs. $76 \%$ and 91 vs. 83 vs. $88 \%$, respectively). This superiority was confirmed and became even more significant in the 10-year update analysis (Fig. 2) [15]. Thus, eight cycles

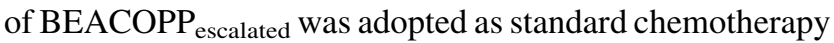
for advanced HL within the GHSG and an increasing number of other cooperative groups. Additional localized RT was applied to residual lymphoma larger than $1.5 \mathrm{~cm}$.

The randomized GHSG HD15 trial aimed at reducing treatment toxicity without compromising efficacy [16]. Chemotherapy within this trial consisted of eight cycles of $\mathrm{BEACOPP}_{\text {escalated, }}$, six cycles of BEACOPP escalated $_{\text {or eight }}$ cycles of BEACOPP-14, a time-dense variant of the $\mathrm{BEACOPP}_{\text {baseline }}$ protocol. Additional localized RT was only applied to patients who had PET-positive residual lymphoma larger than $2.5 \mathrm{~cm}$ after the end of chemotherapy. At 5 years, FFTF rates with eight cycles of BEA-

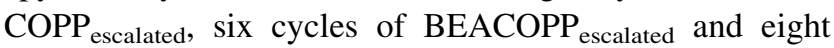
cycles of BEACOPP-14 were $84.4,89.3$ and $85.4 \%$; OS rates were $91.9,95.3$ and $94.5 \%$, respectively. This

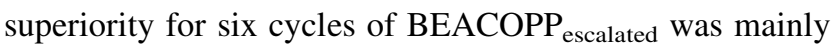
attributed to the lower rate of treatment-related adverse events and fewer deaths due to secondary neoplasia (Table 2). The negative predictive value for the PET was excellent with $94.1 \%$ at 12 months. Thus, six cycles of escalated BEACOPP followed by localized RT of PETpositive residual lymphoma larger than $2.5 \mathrm{~cm}$ became the novel standard of care for the treatment of advanced HL within the GHSG. With this approach, only $11 \%$ of patients still require RT after chemotherapy.

Ongoing studies including patients with advanced HL aim at tailoring treatment according to early interim PET. Two different strategies are evaluated. Some groups such as the GITIL (Gruppo Italiano Terapie Innovative nei Linfomie) start with ABVD chemotherapy and intensify treatment in case of no complete metabolic response in the interim PET. Other groups such as the GHSG start with the

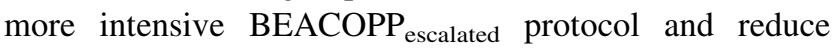
treatment in patients with a negative interim PET. In the GITIL HD0607 trial (NCT00795613), all patients initially receive two cycles of ABVD. Then, interim PET is performed. Patients with a negative PET continue treatment with ABVD, patients with PET-positive residual disease switch to the BEACOPP escalated $_{\text {protocol. After four cycles }}$ of either ABVD or BEACOPP escalated, another PET is performed. Depending on the result, further treatment procedures vary between observation and allogeneic stem cell transplantation (aSCT). Within the GHSG HD18 trial (NCT00515554), patients initially receive two cycles of

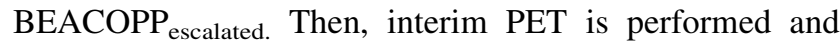
patients are randomized. The standard arm consists of a total of six cycles of BEACOPP escalated $_{\text {irrespective of the }}$ result of the interim PET. In the experimental treatment arm, patients with a complete metabolic response receive a

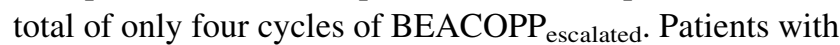
PET-positive residual disease after two cycles of chemotherapy received a total of six cycles of escalated BEACOPP supplemented by the anti-CD20 antibody rituximab in the cycles applied after the interim PET until this study arm was recently closed according to plan.

Since results with current approaches are difficult to improve, future studies will probably aim at developing more personalized treatment strategies in order to spare treatment whenever possible. In addition to interim PET, biomarkers such as the thymus and activation-regulated chemokine (TARC), the number of CD68-positive macrophages in the tumor tissue or others might be included in a score predicting the individual patient's risk [17-20]. Furthermore, treatment could be optimized by combining conventional chemotherapy with novel targeted and less toxic drugs. Ongoing phase I/II studies such as the GHSG "Targeted BEACOPP" study (NCT01569204) combining a BEACOPP backbone with the antibody-drug conjugate (ADC) brentuximab vedotin (SGN-35) currently evaluate such combinations in terms of activity, feasibility and toxicity.

Despite these recent developments, the question whether ABVD or BEACOPP escalated should be the standard of care in the first-line treatment of advanced HL is still being discussed controversially.

\section{Treatment of relapsed HL}

The standard treatment for the majority of patients with relapsed HL consists in high-dose chemotherapy followed by autologous stem cell transplantation (ASCT). Two randomized clinical trials showed the superiority of this 
approach in comparison with conventional chemotherapy. The first trial came from British National Lymphoma Investigation (BNLI) [21]. Patients with relapsed HL were randomly assigned to receive high-dose BEAM (BCNU, etoposide, ara-C, melphalan) followed by autologous bone marrow transplantation (ABMT) or the same chemotherapy protocol at a lower dose without subsequent autograft. Tumor control was better among patients who received high-dose chemotherapy followed by ABMT resulting in significantly better EFS and progression-free survival (PFS) rates. However, OS did not significantly differ between study arms. The second, larger study was conducted by the GHSG and the Lymphoma Working Party of the European Group for Blood and Marrow Transplantation (EBMT) [22]. All 161 patients included initially received two cycles of Dexa-BEAM. Chemosensitive patients were then randomized between two additional cycles of the same chemotherapy or high-dose BEAM followed by ASCT. At a median follow-up of 39 months, 3-year FFTF was superior for patients receiving high-dose chemotherapy and ASCT. However, similar to the British trial, OS did not significantly differ between patient groups.

In the follow-up trial conducted by the GSHG, the EORTC, the EBMT and the Spanish Grup per l'Estudi dels Linfomes de Catalunya i Balears (GELCAB), all patients enrolled initially received two cycles of DHAP (dexamethasone, high-dose ara-C, cisplatin) [23]. Patients responding to DHAP were randomized between high-dose BEAM followed by ASCT and a sequential high-dose program consisting of cyclophosphamide, methotrexate/ vincristine, etoposide and BEAM followed by ASCT. At a median observation of 42 months, no differences between treatment arms in terms of FFTF and OS were detectable. Thus, the less toxic approach consisting of two cycles of DHAP or other salvage protocols such as IGEV (ifosphamide, gemcitabine, etoposide, vinblastine) and ICE (ifosphamide, carboplatin, etoposide) followed by high-dose chemotherapy and ASCT remains standard of care in the treatment of patients with relapsed HL [24, 25].

Ongoing trials aim at optimizing salvage therapy by combining established re-induction protocols with novel drugs such as antibodies or small molecules on the one hand and aim at identifying risk groups by the use of interim PET on the other hand. Outcome of high-risk patients may be improved by maintenance therapy, an approach that is currently evaluated.

\section{Treatment of elderly patients with HL}

HL patients aged 60 years or older are considered elderly. Treatment of this age group represents a challenge since outcome is significantly worse than in younger patients
[26]. This is mainly due to comorbidity and an increased rate of treatment-related toxic events precluding aggressive treatment in many cases. Thus, novel strategies are needed. Several groups recently evaluated approaches particularly developed for this age group. These approaches aimed at being equally or more effective and less toxic than the ABVD regimen which is considered standard of care although increased toxicity was also observed with this protocol in elderly patients [27]. The GHSG conducted a phase II study including 59 patients with early unfavorable and advanced HL aged 60-75 years. Patients were treated with 6-8 cycles of the PVAG (prednisone, vinblastine, adriamycin, gemcitabine) regimen optionally followed by RT [28]. Response rates were comparable to those observed with ABVD, toxicity was acceptable. At a median follow-up of 37 months, 3-year PFS and OS rates were 58 and $66 \%$, respectively. These results justify further evaluation of this protocol. The Scotland and Newcastle Lymphoma Group (SNLG) recently published results of a study investigating the VEPEMB (vinblastine, cyclophosphamide, prednisone, procarbazine, etoposide, mitoxantrone, bleomycin) regimen in 103 elderly HL patients. With this protocol, 3-year PFS rates for patients with early and advanced HL were 74 and $58 \%$, 3-year OS rates were 80.7 and $66.2 \%$, respectively. Thus, this regimen might also represent an option in elderly HL patients [29]. An ongoing phase I study by the GHSG combines AVD with lenalidomide (NCT01056679). Bleomycin is not given to reduce the risk for pulmonary toxicity. However, results from this trial are pending. In case of relapse, treatment of elderly HL patients is mostly palliative since only few patients from this age group are candidates for high-dose chemotherapy followed by ASCT. In the majority of cases, single-agent chemotherapy with drugs such as gemcitabine is given [30]. Alternatively, patients can be included in phase I/II studies evaluating novel targeted drugs.

\section{Treatment of nodular lymphocyte-predominant HL (NLPHL)}

Nodular lymphocyte-predominant HL (NLPHL) is a rare subtype representing about $5 \%$ of HL cases [1]. When compared with classical HL (cHL), NLPHL is characterized by a more indolent clinical course often resembling indolent B cell non-Hodgkin lymphoma (NHL) and a substantially different immunophenotype with consistent positivity for CD20 [31, 32]. Due to its mostly indolent clinical course, the standard treatment for early favorable NLPHL recommended by most cooperative groups consists of RT alone [33, 34]. Results with this approach are excellent with remission rates close to $100 \%$ and longterm survival in about $95 \%$ [35]. A recent GHSG study 
evaluated the anti-CD20 antibody rituximab in the treatment of stage IA NLPHL patients with the aim to reduce the risk of long-term toxicity such as RT-associated secondary solid tumors [36]. Patients received four weekly standard doses $\left(375 \mathrm{mg} / \mathrm{m}^{2}\right)$ of the antibody. All 28 patients included in the study responded to treatment. However, relapse rate at a median follow-up of 43 months was $25 \%$ and thus significantly higher than with RT only so that this approach cannot be recommended for the majority of patients with early favorable NLPHL. Early unfavorable and advanced NLPHL are usually treated very similar to cHL since no mature data on alternative treatment approaches for NLPHL are available to date. In relapsed NLPHL, the standard of care for cHL consisting of high-dose chemotherapy followed by ASCT appears to be less effective resulting in higher relapse rates [37]. Thus, anti-CD20 antibody treatment represents an alternative in this situation. A study including 15 patients with relapsed NLPHL reported a response rate of $94 \%$ and durable remissions in a relevant proportion of patients after treatment with rituximab [38]. Given the low toxicity of the antibody, rituximab can therefore be considered treatment of choice in relapsed NLPHL, at least in patients with more localized disease. An ongoing trial by the GHSG evaluates the tolerability and efficacy of the fully human secondgeneration anti-CD20 antibody ofatumumab in relapsed NLPHL (NCT01187303).

\section{Novel drugs}

Despite the high cure rates achieved with first-line and second-line approaches, treatment of patients with relapse after high-dose chemotherapy and ASCT still represents a field of unmet medical need since most of these patients finally succumb to their disease [39]. Thus, a plethora of novel targeted drugs including antibodies, small molecules and ADC were evaluated in recent years to improve treatment of this patient group. The most advanced of these drugs is the ADC brentuximab vedotin consisting of the synthetic chemotherapeutic agent monomethyl-auristatin $\mathrm{E}$ (MMAE) linked to an anti-CD30 antibody (Fig. 3). After efficacy was shown in a phase I trial including 45 patients with relapsed and refractory CD30-positive hematologic malignancies, a pivotal trial with $102 \mathrm{HL}$ patients who had relapsed after high-dose chemotherapy and ASCT was conducted $[40,41]$. Patients received brentuximab vedotin at a dose of $1.8 \mathrm{mg} / \mathrm{kg}$ every 3 weeks up to a maximum of 16 cycles. The overall response rate (ORR) reported was $75 \%$ with CR in $34 \%$ of patients. These excellent data led to the approval of the drug in the US and in Europe. A report by the GHSG on patients with multiple relapses treated within a named patient program confirmed the excellent efficacy and tolerability of brentuximab vedotin [42]. Additional analyses on the use prior to aSCT, at relapse after aSCT and in transplant-naïve patients extend
Fig. 3 Mechanism of action of the antibody-drug conjugate brentuximab vedotin (SGN-35) (1 ADC binds to CD30, 2 ADCCD30 complex traffics to lysosome, 3 MMAE is released, 4 MMAE disrupts microtubule network, $5 \mathrm{G} 2 / \mathrm{M}$ cell cycle arrest, 6 apoptosis)

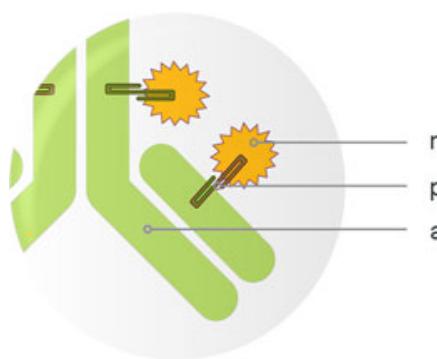

monomethyl auristatin $\mathrm{E}$ (MMAE), potent antitubulin agent
protease-cleavable linker
anti-CD30 monoclonal antibody

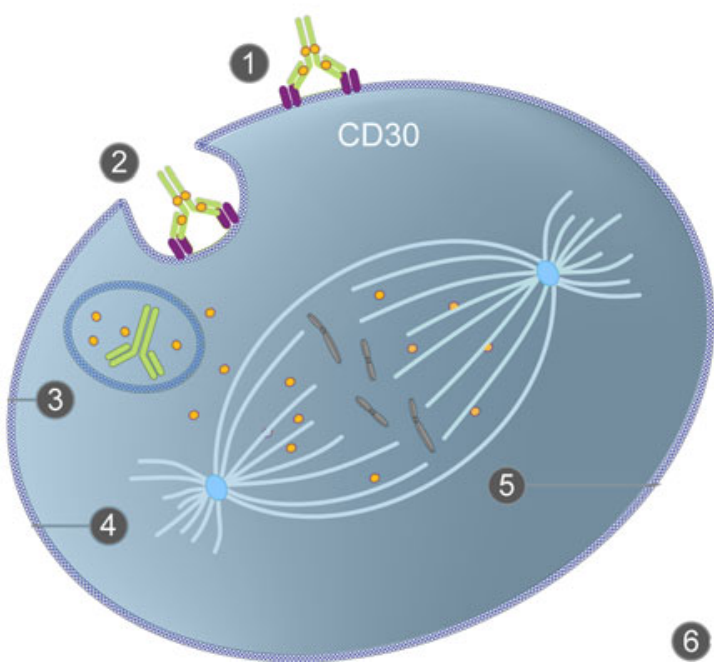


the promising findings on brentuximab vedotin [43-45]. Other novel drugs that were recently shown to be active in HL include the immunomodulatory drug lenalidomide, the mTOR inhibitor everolimus and HDAC inhibitor panobinostat [46-49]. However, all of them appear to be either less effective or more toxic or both when compared with brentuximab vedotin. Nonetheless, they might also have a role in the treatment of HL, either alone or in combination with conventional chemotherapy.

\section{References}

1. Eichenauer DA, Engert A, Dreyling M. Hodgkin's lymphoma: ESMO clinical practice guidelines for diagnosis, treatment and follow-up. Ann Oncol. 2011;22 Suppl 6:vi55-8.

2. Engert A, Franklin J, Eich HT, Brillant C, Sehlen S, Cartoni C, et al. Two cycles of doxorubicin, bleomycin, vinblastine, and dacarbazine plus extended-field radiotherapy is superior to radiotherapy alone in early favorable Hodgkin's lymphoma: final results of the GHSG HD7 trial. J Clin Oncol. 2007 Aug 10;25(23):3495-502.

3. Ferme C, Eghbali H, Meerwaldt JH, Rieux C, Bosq J, Berger F, et al. Chemotherapy plus involved-field radiation in early-stage Hodgkin's disease. N Engl J Med. 2007;357(19):1916-27.

4. Engert A, Plutschow A, Eich HT, Lohri A, Dorken B, Borchmann $\mathrm{P}$, et al. Reduced treatment intensity in patients with early-stage Hodgkin's lymphoma. N Engl J Med. 2010;363(7):640-52.

5. Borchmann P, Diehl V, Goergen H, Lohri A, Zijlstra J, Topp M, et al. Dacarbazine is an essential component of ABVD in the treatment of early favourable Hodgkin lymphoma: results of the second interim analysis of the GHSG HD13 trial. Haematologica. 2010;95, Suppl. 2(473):A1146.

6. Eich HT, Diehl V, Gorgen H, Pabst T, Markova J, Debus J, et al. Intensified chemotherapy and dose-reduced involved-field radiotherapy in patients with early unfavorable Hodgkin's lymphoma: final analysis of the German Hodgkin Study Group HD11 trial. J Clin Oncol. 2010;28(27):4199-206.

7. Noordijk EM, Thomas J, Ferme C, van't Veer MB, Brice P, Divine M, et al. First results of the EORTC-GELA H9 randomized trials: the H9-F trial (comparing 3 radiation dose levels) and H9-U trial (comparing 3 chemotherapy schemes) in patients with favorable or unfavorable early stage Hodgkin's lymphoma (HL). ASCO Meeting Abstracts. 2005;23(16 Suppl):6505.

8. von Tresckow B, Plutschow A, Fuchs M, Klimm B, Markova J, Lohri A, et al. Dose-intensification in early unfavorable Hodgkin's lymphoma: final analysis of the German hodgkin study group HD14 trial. J Clin Oncol. 2012;30(9):907-13.

9. Diehl V, Franklin J, Hasenclever D, Tesch H, Pfreundschuh M, Lathan B, et al. BEACOPP, a new dose-escalated and accelerated regimen, is at least as effective as COPP/ABVD in patients with advanced-stage Hodgkin's lymphoma: interim report from a trial of the German Hodgkin's Lymphoma Study Group. J Clin Oncol. 1998;16(12):3810-21.

10. Meyer RM, Gospodarowicz MK, Connors JM, Pearcey RG, Wells WA, Winter JN, et al. ABVD alone versus radiation-based therapy in limited-stage Hodgkin's lymphoma. N Engl J Med. 2012;366(5):399-408.

11. Canellos GP, Anderson JR, Propert KJ, Nissen N, Cooper MR, Henderson ES, et al. Chemotherapy of advanced Hodgkin's disease with MOPP, ABVD, or MOPP alternating with ABVD. N Engl J Med. 1992;327(21):1478-84.
12. Canellos GP, Niedzwiecki D. Long-term follow-up of Hodgkin's disease trial. N Engl J Med. 2002;346(18):1417-8.

13. Canellos GP, Niedzwiecki D, Johnson JL. Long-term follow-up of survival in Hodgkin's lymphoma. $N$ Engl $J$ Med. 2009;361(24):2390-1.

14. Diehl V, Franklin J, Pfreundschuh M, Lathan B, Paulus U, Hasenclever D, et al. Standard and increased-dose BEACOPP chemotherapy compared with COPP-ABVD for advanced Hodgkin's disease. N Engl J Med. 2003;348(24):2386-95.

15. Engert A, Diehl V, Franklin J, Lohri A, Dorken B, Ludwig WD, et al. Escalated-dose BEACOPP in the treatment of patients with advanced-stage Hodgkin's lymphoma: 10 years of follow-up of the GHSG HD9 study. J Clin Oncol. 2009;27(27):4548-54.

16. Engert A, Haverkamp H, Kobe C, Markova J, Renner C, Ho A, et al. Reduced-intensity chemotherapy and PET-guided radiotherapy in patients with advanced stage Hodgkin's lymphoma (HD15 trial): a randomised, open-label, phase 3 non-inferiority trial. Lancet. 2012;379(9828):1791-9.

17. Weihrauch MR, Manzke O, Beyer M, Haverkamp H, Diehl V, Bohlen $\mathrm{H}$, et al. Elevated serum levels of $\mathrm{CC}$ thymus and activation-related chemokine (TARC) in primary Hodgkin's disease: potential for a prognostic factor. Cancer Res. 2005;65(13):5516-9.

18. Plattel WJ, van den Berg A, Visser L, van der Graaf AM, Pruim J, Vos $\mathrm{H}$, et al. Plasma thymus and activation-regulated chemokine as an early response marker in classical Hodgkin's lymphoma. Haematologica. 2012;97(3):410-5.

19. Steidl C, Lee T, Shah SP, Farinha P, Han G, Nayar T, et al. Tumor-associated macrophages and survival in classic Hodgkin's lymphoma. N Engl J Med. 2010;362(10):875-85.

20. Tan KL, Scott DW, Hong F, Kahl BS, Fisher RI, Bartlett NL, et al. Tumor-associated macrophages predict inferior outcomes in classical Hodgkin lymphoma: a correlative study from the E2496 Intergroup trial. Blood. 2012 Sep 4.

21. Linch DC, Winfield D, Goldstone AH, Moir D, Hancock B, McMillan A, et al. Dose intensification with autologous bonemarrow transplantation in relapsed and resistant Hodgkin's disease: results of a BNLI randomised trial. Lancet. 1993;341(8852): 1051-4.

22. Schmitz N, Pfistner B, Sextro M, Sieber M, Carella AM, Haenel $\mathrm{M}$, et al. Aggressive conventional chemotherapy compared with high-dose chemotherapy with autologous haemopoietic stem-cell transplantation for relapsed chemosensitive Hodgkin's disease: a randomised trial. Lancet. 2002;359(9323):2065-71.

23. Josting A, Muller H, Borchmann P, Baars JW, Metzner B, Dohner $\mathrm{H}$, et al. Dose intensity of chemotherapy in patients with relapsed Hodgkin's lymphoma. J Clin Oncol. 2010;28(34): 5074-80.

24. Santoro A, Magagnoli M, Spina M, Pinotti G, Siracusano L, Michieli $\mathrm{M}$, et al. Ifosfamide, gemcitabine, and vinorelbine: a new induction regimen for refractory and relapsed Hodgkin's lymphoma. Haematologica. 2007;92(1):35-41.

25. Moskowitz CH, Nimer SD, Zelenetz AD, Trippett T, Hedrick EE, Filippa DA, et al. A 2-step comprehensive high-dose chemoradiotherapy second-line program for relapsed and refractory Hodgkin disease: analysis by intent to treat and development of a prognostic model. Blood. 2001;97(3):616-23.

26. Engert A, Ballova V, Haverkamp H, Pfistner B, Josting A, Duhmke E, et al. Hodgkin's lymphoma in elderly patients: a comprehensive retrospective analysis from the German Hodgkin's Study Group. J Clin Oncol. 2005;23(22):5052-60.

27. Boll B, Gorgen H, Fuchs M, von Tresckow B, Engert A, Borchmann P. Feasibility and efficacy of ABVD in elderly Hodgkin lymphoma patients: analysis of two randomized prospective multicenter trials of the German Hodgkin Study Group (HD10 and HD11). ASH annual meeting abstracts. 2010 November 19 , 2010;116(21):418. 
28. Boll B, Bredenfeld H, Gorgen H, Halbsguth T, Eich HT, Soekler $\mathrm{M}$, et al. Phase 2 study of PVAG (prednisone, vinblastine, doxorubicin, gemcitabine) in elderly patients with early unfavorable or advanced stage Hodgkin lymphoma. Blood. 2011;118(24):6292-8.

29. Proctor SJ, Wilkinson J, Jones G, Watson GC, Lucraft HH, Mainou-Fowler T, et al. Evaluation of treatment outcome in 175 patients with Hodgkin lymphoma aged 60 years or over: the SHIELD study. Blood. 2012;119(25):6005-15.

30. Santoro A, Bredenfeld H, Devizzi L, Tesch H, Bonfante V, Viviani $S$, et al. Gemcitabine in the treatment of refractory Hodgkin's disease: results of a multicenter phase II study. J Clin Oncol. 2000;18(13):2615-9.

31. Nogova L, Rudiger T, Engert A. Biology, clinical course and management of nodular lymphocyte-predominant hodgkin lymphoma. Hematology Am Soc Hematol Educ Program. 2006;2006:266-72.

32. Nogova L, Reineke T, Brillant C, Sieniawski M, Rudiger T, Josting A, et al. Lymphocyte-predominant and classical Hodgkin's lymphoma: a comprehensive analysis from the German Hodgkin Study Group. J Clin Oncol. 2008;26(3):434-9.

33. Nogova L, Reineke T, Eich HT, Josting A, Muller-Hermelink HK, Wingbermuhle K, et al. Extended field radiotherapy, combined modality treatment or involved field radiotherapy for patients with stage IA lymphocyte-predominant Hodgkin's lymphoma: a retrospective analysis from the German Hodgkin Study Group (GHSG). Ann Oncol. 2005;16(10):1683-7.

34. Hoppe RT, Advani RH, Ai WZ, Ambinder RF, Aoun P, Bello CM, et al. Hodgkin lymphoma, version 2.2012 featured updates to the NCCN guidelines. J Natl Compr Canc Netw. 2012;10(5):589-97.

35. Chen RC, Chin MS, Ng AK, Feng Y, Neuberg D, Silver B, et al. Early-stage, lymphocyte-predominant Hodgkin's lymphoma: patient outcomes from a large, single-institution series with long follow-up. J Clin Oncol. 2010;28(1):136-41.

36. Eichenauer DA, Fuchs M, Pluetschow A, Klimm B, Halbsguth T, Boll B, et al. Phase 2 study of rituximab in newly diagnosed stage IA nodular lymphocyte-predominant Hodgkin lymphoma: a report from the German Hodgkin Study Group. Blood. 2011;118(16):4363-5.

37. Jackson C, Sirohi B, Cunningham D, Horwich A, Thomas K, Wotherspoon A. Lymphocyte-predominant Hodgkin lymphoma-clinical features and treatment outcomes from a 30-year experience. Ann Oncol. 2010;21(10):2061-8.

38. Schulz H, Rehwald U, Morschhauser F, Elter T, Driessen C, Rudiger $\mathrm{T}$, et al. Rituximab in relapsed lymphocyte-predominant Hodgkin lymphoma: long-term results of a phase 2 trial by the
German Hodgkin Lymphoma Study Group (GHSG). Blood. 2008;111(1):109-11.

39. Horning S, Fanale M, De Vos S, Borchmann P, Illidge T, Engert A, et al. Defining a population of Hodgkin lymphoma patients for novel therapeutics: an international effort. Ann Oncol 2008;19(Suppl 4) (June):abstract 118.

40. Younes A, Bartlett NL, Leonard JP, Kennedy DA, Lynch CM, Sievers EL, et al. Brentuximab vedotin (SGN-35) for relapsed CD30-positive lymphomas. N Engl J Med. 2010;363(19): 1812-21.

41. Younes A, Gopal AK, Smith SE, Ansell SM, Rosenblatt JD, Savage KJ, et al. Results of a pivotal phase II study of brentuximab vedotin for patients with relapsed or refractory Hodgkin's lymphoma. J Clin Oncol. 2012;30(18):2183-9.

42. Rothe A, Sasse S, Goergen H, Eichenauer DA, Lohri A, Jager U, et al. Brentuximab vedotin for relapsed or refractory CD30+ hematologic malignancies: the German Hodgkin Study Group experience. Blood. 2012;120(7):1470-2.

43. Chen R, Palmer JM, Thomas SH, Tsai NC, Farol L, Nademanee A, et al. Brentuximab vedotin enables successful reduced-intensity allogeneic hematopoietic cell transplantation in patients with relapsed or refractory Hodgkin lymphoma. Blood. 2012;119(26): 6379-81.

44. Gopal AK, Ramchandren R, O'Connor OA, Berryman RB, Advani RH, Chen R, et al. Safety and efficacy of brentuximab vedotin for Hodgkin lymphoma recurring after allogeneic stem cell transplantation. Blood. 2012;120(3):560-8.

45. Forero-Torres A, Fanale M, Advani R, Bartlett NL, Rosenblatt JD, Kennedy DA, et al. Brentuximab vedotin in transplant-naive patients with relapsed or refractory Hodgkin lymphoma: analysis of two phase I studies. Oncologist. 2012;17(8):1073-80.

46. Boll B, Borchmann P, Topp MS, Hanel M, Reiners KS, Engert A, et al. Lenalidomide in patients with refractory or multiple relapsed Hodgkin lymphoma. Br J Haematol. 2010;148(3):480-2.

47. Fehniger TA, Larson S, Trinkaus K, Siegel MJ, Cashen AF, Blum $\mathrm{KA}$, et al. A phase 2 multicenter study of lenalidomide in relapsed or refractory classical Hodgkin lymphoma. Blood. 2011;118(19):5119-25.

48. Johnston PB, Inwards DJ, Colgan JP, Laplant BR, Kabat BF, Habermann TM, et al. A phase II trial of the oral mTOR inhibitor everolimus in relapsed Hodgkin lymphoma. Am J Hematol. 2010;85(5):320-4.

49. Younes A, Sureda A, Ben-Yehuda D, Zinzani PL, Ong TC, Prince HM, et al. Panobinostat in patients with relapsed/refractory Hodgkin's lymphoma after autologous stem-cell transplantation: results of a phase II study. J Clin Oncol. 2012;30(18):2197-203. 